\title{
El trastorno por estrés post-traumático relacionado con el combate entre los veteranos croatas: modelos causales para grupos (clusters) de síntomas
}

\author{
Željka Vukšić-Mihaljević, PhD \\ Mirta Benšić \\ Dražen Begić \\ Gordan Lauc \\ Bernarda Hutinec \\ Vladimir Čandrlić \\ Verica Todorović
}

Department of Psychiatry University Hospital Osijek, University School of Medicine

CROATIA

RESUMEN - Objetivos: Este estudio investiga el papel etiológico de los factores de riesgo premilitares, las condiciones de entrada en el ejército, las experiencias en zona de guerra, reacciones disociativas y el recibimiento al regreso al hogar en el desarrollo de grupos (clusters) de síntomas de trastorno por estrés postraumático (TEP) crónico entre los veteranos croatas.

Métodos: Se seleccionó una muestra de 150 veteranos de guerra croatas con diagnóstico de TEP crónico relacionado con el combate que solicitaron tratamiento en el Departamento de Psiquiatría del Hospital Universitario de Osijek, Croacia, que proporcionaron los datos completos, para realizar este estudio entre los ex-soldados que solicitaron tratamiento $(\mathrm{N}=192)$. Se utilizaron modelos de ecuaciones estructurales para desarrollar un modelo etiológico que estudiase las relaciones entre factores de riesgo premilitar, condiciones de entrada en el ejercito, experiencias en zona de guerra, reacciones disociativas, recibimiento al regreso al hogar y los grupos (“clusters”) de síntomas presentes de TEP.

Resultados: Se desarrollaron los modelos causales con satisfactorios ajuste y dimensiones. El análisis de resultados sugiere que, en los tres modelos estudiados, hay un diferente efecto etiológico de las variables estudiadas sobre los clusters de síntomas TEP. En los tres modelos estudiados, las experiencias en zona de guerra, la disociación peritraumática y el recibimiento al regreso al hogar, tienen un mayor efecto etiológico primario que el derivado de los factores de riesgo premilitares y las condiciones de entrada en el ejército. En ocasiones, se observa un mayor efecto etiológico de los factores de riesgo premilitares en los modelos causales para los síntomas de evitación y las condiciones de entrada en el ejército en los modelos causales para el cluster de activación.

Conclusiones: Los resultados pueden apoyar la hipótesis del estudio de que todos los síntomas de TEP no tienen la misma etiología, y que existe una jerarquización de influencia etiológica diferente entre las variables estudiadas en los tres modelos desarrollados de clusters de síntomas de TEP. 


\section{Introducción}

Está surgiendo un acuerdo general sobre la opinión de que algunos acontecimientos traumáticos y no traumáticos juegan un papel etiológico en el trastorno por estrés postraumático (TEP) y que la tarea más crítica es articular la red de conexiones entre ellos. Los estudios sobre TEP han utilizado casi invariablemente un solo índice global de sintomatología del TEP, en lugar de diferenciar los tres grupos de síntomas DSM-IV. Basarse en un solo índice de sintomatología lleva implícito asumir que todos los síntomas del TEP tienen la misma etiología, pero dicha asunción podría no ser correcta (Bryant \& Harvey 1996, Kulka et al. 1990, Fontana \& Rosenheck 1993).

Nosotros examinamos un modelo causal de grupos de síntomas de TEP en los veteranos de guerra croatas mediante modelos de ecuaciones estructurales (MEE), para determinar las contribuciones relativas de diferentes categorías de experiencia (factores de riesgo premilitares, condiciones de entrada en el ejército, experiencias en zona de guerra, reacciones disociativas a las experiencias en zona de guerra, recibimiento al regreso al hogar) para el desarrollo de grupos de síntomas de TEP. Los MEE son un conjunto de procedimientos estadísticos que determinan las bases o fundamentos de las relaciones subyacentes entre las variables observadas (Bollen 1989).

Nuestra hipótesis es que la experiencia en zona de guerra contribuye más al desarrollo del TEP que los factores de riesgo premilitares, las condiciones de entrada en el ejército, los síntomas disociativos peritraumáticos y el recibimiento al regreso al hogar.

\section{Método}

\section{Selección de la muestra}

La muestra de sujetos, que incluye a 150 pacientes ambulatorios que solicitaron tratamiento, se seleccionó de entre los pacientes $(\mathrm{N}=192)$ con un diagnóstico de TEP crónico relacionado con el combate según la CIE-10 (establecido en un tratamiento psiquiátrico previo por psiquiatras clínicos), que fueron tratados en el Departamento de Psiquiatría del Hospital Clínico de Osijek, Croacia, en los cinco años previos. El período de inclusión en el estudio fue de un mes. La "puerta" de entrada al estudio era ser paciente en control ambulatorio por trauma psicológico. En el momento del estudio, todos los sujetos cumplían plenamente criterios para TEP según la Entrevista Clínica Estructurada para el DSM-IV (sección TEP) (First et al. 2002), aplicada por psiquiatras. Los pacientes eran incluídos en el estudio si eran hombres, veteranos de guerra, entre 30 y 45 años, sin evidencia obvia de síndrome orgánico cerebral, retraso mental, dependencia del alcohol o psicosis, según el DSM-IV. Los pacientes eran incluídos si habían estado en combate directo o habían desempeñado cargos con alto grado de estrés de combate, por un mínimo de tres meses consecutivos durante la Guerra en Croacia (1991-1996). Todos los sujetos dieron el consentimiento informado para participar en el estudio. Cuarenta y dos pacientes no entraron en el estudio $(n=16$ por TEP incompleto, $\mathrm{n}=12$ por dependencia del alcohol grave, $n=4$ por evidencia obvia de retraso mental, $\mathrm{n}=4$ por síndrome orgánico cerebral, $\mathrm{n}=1$ trastorno psicótico y $\mathrm{n}=5$ por no dar el consentimiento informado). 


\section{Variables demográficas e historia psicosocial}

Los sujetos en el estudio tenían una edad media de 37,5 $\pm 5,9$ años. El nivel educacional de la muestra era: escuela elemental incompleta $(n=8)$, escuela elemental completa $(n=28)$, escuela primaria incompleta $(n$ $=46)$, escuela primaria completa $(n=68)$. El estado civil de la muestra en el momento de la evaluación era: casado $(\mathrm{n}=118)$, soltero $(\mathrm{n}$ $=20)$ y divorciado $(n=12)$. Respecto a la situación laboral, se incluían: empleado $(\mathrm{n}=$ $40)$, desempleado $(n=6)$, jubilado $(n=28)$ y bajo el cuidado del Departamento Militar para la Atención Médico-Social $(n=76)$. En cuanto a la paternidad, 121 sujetos tenían uno o más hijos.

\section{Estresores militares}

Todos los sujetos eran soldados de ejército croata durante la Guerra en Croacia (1991-1996), lo que justificaba un perfil de factores estresantes del combate. Cincuenta de los veteranos habían sido heridos en combate.

\section{Determinaciones}

Los datos se obtuvieron mediante una batería de instrumentos estandarizados e ítems especialmente construídos (dos secciones de entrevista estructurada y una sección de la encuesta de auto-informe). La entrevista fue administrada por médicos. Los datos fueron recogidos retrospectivamente, a excepción de los síntomas. Los conjuntos de variables tenían una clara ordenación histórico-temporal. Nuestra hipótesis es que cada conjunto de variables contribuye causalmente a cada grupo subsiguiente de eventos y experiencias.
Los factores de riesgo premilitar incluyeron las siguientes siete variables:

El abuso físico o sexual en la niñez se midió como una variable dicotómica, de acuerdo con la Lista de Eventos Traumáticos y Abuso Físico Explícito (Rausch \& Knutson 1991) $($ media $=0,30 \pm 0,79)$.

El trastorno de conducta incluía once conductas, indicativas de un trastorno de conducta antes de los 15 años, tomadas de la lista compilada por Helzer et al. (Helzer et al. 1987, Helzer 1981) $($ media $=1,10 \pm 1,80)$.

La inestabilidad familiar se midió como variable dicotómica, utilizando la Escala de Estabilidad Familiar (Kadushin et al. 1981) (media $=0,93 \pm 1,25)$.

El tratamiento u hospitalización por un problema psiquiátrico antes de que el veterano tuviera 18 años se determinó con el ítem dicotómico “¿Ha sido Vd. alguna vez tratado u hospitalizado por un problema psicológico?" (media = 0,02 $\pm 0,14)$.

La hospitalización paternal por un problema psiquiátrico antes de que el veterano cumpliera 18 años se determinó con el ítem dicotómico "¿Alguno de sus padres fue alguna vez hospitalizado por un problema psiquiátrico?" (media $=0,06 \pm 0,24)$.

La exposición del padre al combate se determinó con el ítem dicotómico “ ¿Ha estado su padre alguna vez en la vida implicado en combate como soldado durante la/s guerra/s?" (media $=0,22 \pm 0,41)$.

El nivel educacional se midió como una variable discreta "¿Qué nivel educativo alcanzó Vd. antes de la guerra?". Los niveles educacionales se codificaron de 1 a 5 de manera ascendente $($ media $=3,18 \pm 0,94)$.

Las condiciones de entrada en el ejército incluyen ingreso voluntario y edad en el momento de ingreso. 
A cada veterano se le preguntó cómo entró por primera vez en el ejército, generando una variable dicotómica: a) ingreso voluntario; b) reacio al ingreso (media $=$ $0,95 \pm 0,22$ ).

La edad al ingreso se determinaba en una parte de la entrevista estructurada y se medía como una variable continua (media $=$ $30,24 \pm 6,01)$.

Las experiencias en zona de guerra incluyen las siguientes cuatro variables:

El combate se medía con la Escala de Exposición de Combate (Lee et al. 1995). Una puntuación de combate superior a 6 indicaba un nivel alto de exposición al combate $($ media $=8,62 \pm 1,17)$.

Haber sido testigo y haber participado en violencia abusiva se midió dicotómicamente en la entrevista estructurada (Fontana \& Rosenheck 1993). De acuerdo con Laufer $e t$ al. (1985), codificamos la exposición a violencia abusiva en dos categorías mutuamente excluyentes: sólo siendo testigo del incidente (media $=0,37 \pm 0,81)$ y participación de uno mismo, sin tener en cuenta que otros eran testigos (media $=0,09 \pm 0,35)$.

Haber recibido una acción disciplinaria se codificó dicotómicamente a partir de una pregunta (Fontana \& Rosenheck 1993) que indagaba si el veterano alguna vez recibió alguna acción disciplinaria mientras estaba en el ejército (media $=0,54 \pm 0,28)$.

La disociación era medida en la entrevista estructurada como la media de cinco ítems dicotómicos (Marmar 1989) que describían aspectos críticos de una reacción disociativa (media $=0,54 \pm 0,28)$.

El recibimiento en el regreso al hogar incluía dos variables (Fontana \& Rosenheck 1993). El recibimiento por parte la sociedad se codificó determinando el rechazo de bienvenida, con un rango desde 0 (nada) a 3 (extremadamente malo) (media $=2,00 \pm$ 1,21). El recibimiento familiar al veterano se midió mediante dos escalas. La no disponibilidad de ayuda se codificó determinando el bajo soporte, con un rango desde 0 (nada) hasta 4 (extremadamente) (media $=1,40 \pm$ 1,52). Carecer de alguien para hablar se codificó determinando el bajo soporte, con un rango desde 0 (nada) a 3 (extremadamente) $($ media $=0,89 \pm 1,05)$. Se generó una variable latente de apoyo bajo en el modelo. Su cambio se explica por los ajustes de sus indicadores, poca ayuda $(0,82)$ y poca conversación $(0,65)$.

Los síntomas de TEP se midieron mediante la PTSD checklist (PCL-M), que es una escala de evaluación de TEP autoadministrada (Cura et al. 1993, Blanchard et al. 1996). Se instruyó a los sujetos para que indicasen cuánto les ha molestado cada síntoma en el último mes, utilizando una escala de 5 puntos [desde 1 (nada) hasta 5 (extremadamente)]. Se sumaron las puntuaciones de los 17 síntomas del PCL-M para generar una puntuación continua de la gravedad de los síntomas de TEP (media = $71,47 \pm 7,57)$. Se sumaron las puntuaciones de los clusters de síntomas: cluster B (1-5) $($ media $=21,13 \pm 3,05)$, cluster $\mathrm{C}(6-12)$ (media $=28,71 \pm 3,77)$, y cluster $\mathrm{D}(13-17)$ $($ media $=21,63 \pm 2,56)$.

\section{Análisis de datos}

Los datos en este estudio proceden de un estudio transversal y los informes son retrospectivos. Las variables seleccionadas para incluirlas en los modelos tienen una clara ordenación histórica, que se utilizó como la base lógica para la especificación de los modelos. Construímos tres modelos causales: uno para el cluster de re-experi- 
mentación (TEP B); para el cluster de evitación y entorpecimiento emocional (TEP C); y para el cluster de activación (TEP D).

Una característica de los modelos de ecuaciones estructurales (MEE) es que el error es una parte del componente de la medida, que se estima por separado. Las variables latentes se considera que están desprovistas de error de medida y son, por tanto, absolutamente fiables. En los MEE que utilizan tanto componentes de medida como estructurales, con variables latentes presumible y absolutamente fiables, los pesos de la regresión son las estimaciones imparciales de sus parámetros, una necesidad para la inferencia estadística precisa. Los MEE son unos procedimientos de estimación de información completos, ya que emplean toda la información disponible en la base de datos para derivar las estimaciones de parámetros. Basado en la teoría estadística y el modelo hipotetizado, se computan las estimaciones para reproducirse de forma más exacta la matriz de covarianzas observadas entre todas las variables. Los parámetros estiman qué derivaciones son eficaces: se minimizan sus errores estándar y sus estimaciones se aproximan todo lo posible a los valores verdaderos. El MEE es un método satisfactorio para examinar redes complejas de relaciones y las estrategias disponibles para evaluar el ajuste global de modelos y seleccionar el mejor de entre los modelos que compiten. Se han realizado muchos análisis MEE en los datos para registrar la complejidad de las variables que pueden influenciar el desarrollo de los síntomas del TEP y su gravedad (Bollen 1989, Rey \& Rey 1997).

Los MEE especifican el camino causal que se hipotetiza existe entre las variables exógenas y endógenas, y entre las mismas variables endógenas. En cada modelo, los siete factores de riesgo premilitares son variables exógenas cuya causalidad cae fuera del alcance del modelo. La hipótesis es que estas variables afectan a las condiciones de entrada en el ejército, experiencias en zona de guerra, reacciones disociativas ante los traumas en zona de guerra, apoyo postguerra y los clusters de síntomas de TEP. Ninguna asociación causal entre las variables antes citadas se incluyó en la evaluación estadística del modelo, y pueden encontrarse como componentes de la matriz de correlaciones (Tabla I). Las condiciones de entrada en el ejército, las experiencias en zona de guerra, las reacciones disociativas ante los traumas en zona de guerra, el apoyo postguerra y los clusters de síntomas de TEP son variables endógenas que, según nuestras hipótesis, son consecuencia de una o más de las otras variables del modelo, algunas de las cuales son otras variables endógenas. La variable latente de "apoyo bajo" se generó en los modelos. La estimación del modelo fue realizada por estimación de cuadrados mínimos, según el procedimiento SASCALIS (SAS Institute 1990).

Los modelos causales se generaron independientemente para determinar su influencia total, directa e indirecta, en los siguientes clusters de síntomas de TEP: revivir el acontecimiento traumático; evitación y entorpecimiento; e hiperactivación. Los modelos consistieron en una estimación de la influencia de las variables en el modelo directo, indirecto y total para cada cluster de síntomas del TEP. Utilizamos el método de estimación de cuadrados mínimos. La adecuación del modelo al criterio fue satisfactoria. El criterio de aceptación del modelo tiene valores iguales en todos los modelos: índice de bondad de ajuste 0,95; bondad de ajuste para los grados de libertad 0,90; media de cuadrados residual 0,05 .

El modelo TEP B presentó un 13\% del cluster de síntomas de re-experimentación (varianza endógena $=0,87$ ); el modelo TEP 
C presentó un $15 \%$ de los síntomas de evitación y entorpecimiento emocionales (varianza endógena $=0,85$ ); y el modelo TEP D presentó un $21 \%$ del cluster de síntomas de activación (varianza endógena $=0,79$ ).

La Tabla I presenta las correlaciones bivariantes entre las variables del modelo $(\mathrm{N}=$ $150, r=0,10$ significación de $p<0,05)$.

\section{Resultados}

\section{Los modelos causales de clusters de síntomas de TEP}

\section{Efectos totales, directos e indirectos del} cluster de sintomas de re-experimentación (modelo TEP B).

La Tabla II muestra el efecto de las variables del cluster de síntomas de re-experimentación del modelo TEP B, presentando de manera resumida por columnas los efectos totales, directos e indirectos.

Los resultados del estudio muestran que el mayor efecto total entre todas las variables del estudio en el modelo del cluster de síntomas de "re-experimentación de acontecimiento traumático" (TEP B) tiene una variable de acogida al regreso al hogar, seguido por la disociación, las experiencias en zona de guerra, las condiciones de entrada en el ejército y los factores de entrada en el ejército. Es interesante señalar que todas las variables mencionadas del modelo tenían los efectos totales casi iguales, excepto los factores de riesgo premilitares, que eran más bajos. Entre las experiencias en zona de guerra, el efecto total más alto lo tenía la exposición directa al combate. Hablando de las condiciones de entrada al ejército, la edad tenía un efecto total más alto que el ingreso voluntario, mientras que en los fac- tores de riesgo premilitares, el efecto total más alto lo tenía la inestabilidad de la familia primaria.

Efectos totales, directos e indirectos del cluster de síntomas de evitación y embotamiento emocional (modelo TEP C)

La Tabla II muestra el efecto de las variables del cluster de síntomas de evitación y entorpecimiento emocional del modelo TEP $\mathrm{C}$, presentando de manera resumida por columnas los efectos totales, directos e indirectos.

Entre todas las variables estudiadas en el modelo de TEP C, el efecto total más alto estaba en las experiencias en zona de guerra, seguido por la disociación, la acogida al regreso al hogar, los factores de riesgo premilitares y las condiciones de ingreso en el ejército. El efecto total de las primeras tres variables mencionadas es casi igual. Entre las variables de experiencia en zona de guerra, el efecto total más alto lo obtuvo la adaptación a las experiencias de combate (determinado según las medidas disciplinarias), seguido por ser testigo de violencia, exposición al combate y participación en violencia. Una parte importante del alto efecto total se debía a la disociación peritraumática dentro del desarrollo del cluster de síntomas. El efecto total más alto del modelo venía determinado por la falta de apoyo social, en comparación con el insuficiente apoyo de la familia en el recibimiento al regreso al hogar. Entre los factores de riesgo premilitares, el efecto total más alto lo obtuvo haber recibido los sujetos tratamiento anterior debido a sus trastornos psiquiátricos, seguido por la experiencia en guerra del padre, la inestabilidad familiar y los trastornos de conducta, etc. Las condiciones de ingreso en el ejército tienen el efecto total más bajo en este cluster de síntomas, mientras que la 


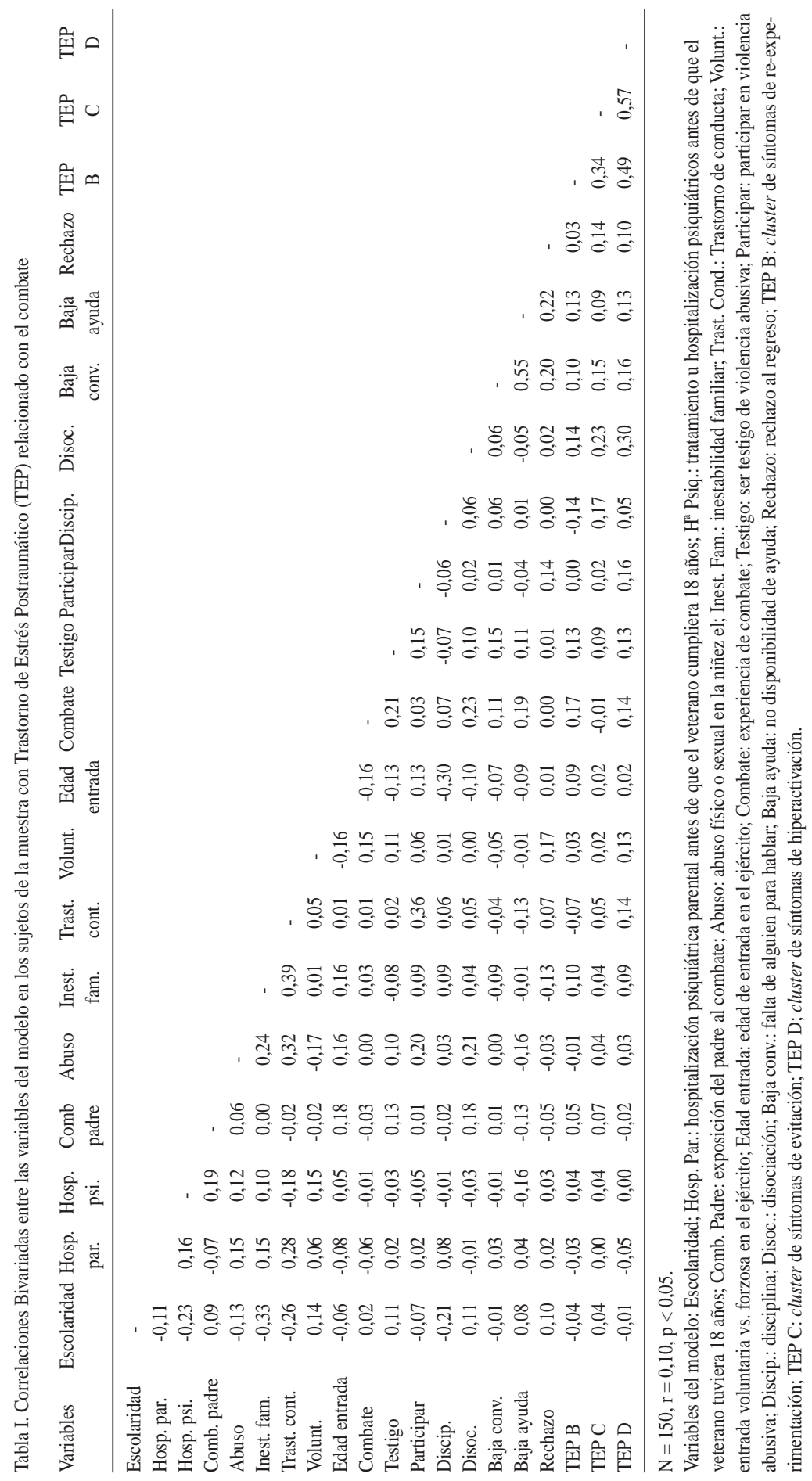


Tabla II

Efectos totales, directos e indirectos de los modelos causales de variables para los distintos clusters de síntomas en una muestra de pacientes con Trastorno de Estrés Postraumático (TEP) relacionado con el combate.

\begin{tabular}{|c|c|c|c|c|c|c|c|c|c|}
\hline \multirow{2}{*}{$\begin{array}{l}\text { Modelos } \\
\text { Efectos }\end{array}$} & \multicolumn{3}{|c|}{$\begin{array}{l}\text { Cluster de } \\
\text { re-experimentación }\end{array}$} & \multicolumn{3}{|c|}{$\begin{array}{l}\text { Cluster de } \\
\text { evitación }\end{array}$} & \multicolumn{3}{|c|}{$\begin{array}{c}\text { Cluster de } \\
\text { hiperactivación }\end{array}$} \\
\hline & Total & Directo & Indirecto & Total & Directo & Indirecto & Total & Directo & Indirecto \\
\hline \multicolumn{10}{|l|}{$\begin{array}{l}\text { Vulnerabilidades } \\
\text { pre-militares }\end{array}$} \\
\hline Escolaridad & $-0,07$ & $-0,10$ & 0,03 & 0,01 & 0,06 & $-0,05$ & $-0,02$ & $-0,02$ & 0,00 \\
\hline Hosp. Par. & 0,03 & 0,02 & 0,01 & 0,09 & 0,09 & 0,00 & 0,03 & 0,03 & 0,00 \\
\hline $\mathrm{H}^{\mathrm{a}}$ psiq. & 0,00 & 0,00 & 0,00 & $-0,04$ & $-0,04$ & 0,00 & $-0,11$ & $-0,11$ & 0,00 \\
\hline Comb. Padre & 0,05 & 0,02 & 0,03 & 0,07 & 0,01 & 0,06 & $-0,01$ & $-0,09$ & 0,08 \\
\hline Abuso & $-0,03$ & $-0,05$ & 0,02 & $-0,01$ & $-0,05$ & 0,04 & $-0,07$ & $-0,07$ & 0,00 \\
\hline Inest. Fam. & 0,16 & 0,15 & 0,01 & 0,05 & 0,04 & 0,01 & 0,05 & 0,04 & 0,01 \\
\hline Trast. Cond. & $-0,13$ & $-0,13$ & 0,00 & 0,05 & 0,06 & $-0,01$ & 0,16 & 0,13 & 0,03 \\
\hline Suma & 0,01 & $-0,09$ & 0,10 & 0,22 & 0,17 & 0,05 & 0,03 & $-0,09$ & 0,12 \\
\hline \multicolumn{10}{|c|}{$\begin{array}{l}\text { Situación de entrada } \\
\text { en el ejército }\end{array}$} \\
\hline Volunt. & 0,03 & 0,02 & 0,01 & 0,01 & 0,02 & $-0,01$ & 0,12 & 0,12 & 0,00 \\
\hline Edad entrada & 0,10 & 0,07 & 0,03 & 0,06 & 0,10 & $-0,04$ & 0,08 & 0,10 & $-0,02$ \\
\hline Suma & 0,13 & 0,09 & 0,04 & 0,07 & 0,12 & $-0,05$ & 0,20 & 0,22 & $-0,02$ \\
\hline \multicolumn{10}{|l|}{$\begin{array}{l}\text { Experiencia en } \\
\text { zona de guerra }\end{array}$} \\
\hline Combate & 0,18 & 0,11 & 0,07 & $-0,02$ & $-0,12$ & 0,10 & 0,11 & $-0,02$ & 0,13 \\
\hline Testigo & 0,11 & 0,10 & 0,01 & 0,11 & 0,10 & 0,01 & 0,12 & 0,09 & 0,03 \\
\hline Participar & 0,00 & 0,00 & 0,00 & $-0,02$ & $-0,02$ & 0,00 & 0,08 & 0,09 & $-0,01$ \\
\hline Discip. & $-0,15$ & $-0,15$ & 0,00 & 0,21 & 0,21 & 0,00 & 0,07 & 0,07 & 0,00 \\
\hline Suma & 0,14 & 0,06 & 0,08 & 0,28 & 0,17 & 0,11 & 0,38 & 0,23 & 0,15 \\
\hline \multicolumn{10}{|l|}{ Disociación } \\
\hline Disoc. & 0,14 & 0,14 & 0,00 & 0,25 & 0,25 & 0,00 & 0,33 & 0,33 & 0,00 \\
\hline \multicolumn{10}{|l|}{$\begin{array}{l}\text { Recepción al } \\
\text { regreso al hogar }\end{array}$} \\
\hline Bajo ароуо & 0,09 & 0,09 & 0,00 & 0,11 & 0,11 & 0,00 & 0,17 & 0,17 & 0,00 \\
\hline Rechazo & 0,06 & 0,03 & 0,03 & 0,14 & 0,11 & 0,03 & 0,00 & 0,00 & 0,00 \\
\hline Suma & 0,15 & 0,12 & 0,03 & 0,25 & 0,22 & 0,03 & 0,17 & 0,17 & 0,00 \\
\hline
\end{tabular}

Criteros de aceptación del modelo: Cluster de re-experimentación: GFI = 0,95; AGFI = 0,90; RMR = 0,05; Cluster de evitación: GFI = 0,95; $\mathrm{AGFI}=0,90 ; \mathrm{RMR}=0,05 ;$ Cluster de hiperactivación: $\mathrm{GFI}=0,95 ; \mathrm{AGFI}=0,90 ; \mathrm{RMR}=0,05$.

Variables del modelo: Escolaridad; Hosp. Par.: hospitalización psiquiátrica parental antes de que el veterano cumpliera 18 años; $\mathrm{H}^{\mathrm{a}}$ Psiq.: tratamiento u hospitalización psiquiátricos antes de que el veterano tuviera 18 años; Comb. Padre: exposición del padre al combate; Abuso: abuso físico o sexual en la niñez el; Inest. Fam.: inestabilidad familiar; Trast. Cond.: Trastorno de conducta; Volunt.: entrada voluntaria vs. forzosa en el ejército; Edad entrada: edad de entrada en el ejército; Combate: experiencia de combate; Testigo: ser testigo de violencia abusiva; Participar: participar en violencia abusiva; Discip.: disciplina; Disoc.: disociación; Bajo apoyo: Bajo apoyo social; Rechazo: rechazo al regreso.

edad juega un papel más importante que el deseo de enrolarse en el ejército. El efecto total relativamente alto de los factores de riesgo premilitares y el mayor efecto total de los problemas de adaptación, entre las variables de experiencias en zona de guerra, podría sugerir cuán importantes son las características de personalidad previa a la guerra en el desarrollo de este cluster de síntomas. 
Efectos totales, directos e indirectos en cluster de síntomas de activación (modelo D de TEP)

La Tabla II muestra el efecto de las variables del cluster de síntomas de activación del modelo TEP D, presentando de manera resumida por columnas los efectos totales, directos e indirectos.

El efecto total más alto de todas las variables estudiadas en el modelo TEP D se observó en las experiencias en zona de guerra, seguido por las disociaciones, las condiciones de entrada en el ejército, el recibimiento al regreso al hogar y los factores de entrada en el ejército. Las experiencias violentas en zona de guerra tenían el efecto total más alto del desarrollo de este cluster, seguido del efecto total de exposición directa al combate. El efecto total de las condiciones de entrada en el ejército era bastante alto para este cluster de síntomas. El efecto total del regreso al hogar en este modelo estaba enfrente de la última variable medida. El efecto total de factores de riesgo premilitares es bastante bajo y, entre ellos, el efecto total más alto fue el derivado de que los sujetos hubiesen padecido un trastorno de conducta antes de los 18 años.

\section{Discusión}

Nuestro análisis sugiere que se da un efecto etiológico diferente de las variables estudiadas sobre los clusters de síntomas de TEP en los tres modelos estudiados. Las experiencias en zona de guerra, la disociación peritraumática y el recibimiento al regreso al hogar tienen un mayor y primario efecto etiológico que los factores de riesgo premilitares y las condiciones de entrada en el ejército, en los modelos estudiados. La excepción es que, a veces, los factores etiológicos de riesgo premilitares son algo más altos en el modelo causal para el cluster de síntomas de evitación y que las condiciones de entrada en el ejército en el modelo causal para el cluster de activación. Los resultados pueden apoyar la hipótesis del estudio de que todos los síntomas de TEP no tienen la misma etiología, y que existe una jerarquía etiológica diferente de influencia entre las variables estudiadas en los modelos de clusters de síntomas de TEP. La etiología de los clusters de síntomas de TEP relacionados con el combate entre los veteranos croatas obtenidos mediante MEE no está suficientemente explicada. La base para esta conclusión es que los modelos explican el $13 \%$, $15 \%$ y $21 \%$ de la varianza para los clusters de intrusividad, evitación y activación. Este estudio presenta la primera aplicación de los MEE en la etiología de los clusters de síntomas de TEP relacionados con el combate entre los veteranos de guerra croatas. Se lograron altos niveles de adecuación de los modelos. Los modelos se basan en asunciones causales que son replicables y no redundantes. Un reciente estudio sobre estructura del síntoma y comorbilidad psiquiátrica entre los veteranos de guerra croatas con TEP (Vukšić-Mihaljević et al. 1999), el estudio de un modelo causal de TEP relacionado con el combate entre los veteranos de guerra croatas (Vukšić-Mihaljević et al. 2000) y los resultados de nuestro estudio señalan que existe heterogeneidad clínica y etiológica del TEP relacionado con el combate. Como ocurre con gran parte de la investigación actual sobre TEP, la validez de los resultados de este estudio podría estar comprometida por el uso de una muestra de conveniencia y por la fiabilidad de la valoración retrospectiva en un momento puntual de variables clave. 
Hemos hallado un alto efecto etiológico de las experiencias en zona de guerra entre las variables estudiadas en todos los modelos de clusters de síntomas de TEP. La especificidad de varios estresores de la zona de guerra para los diferentes síntomas de TEP se describe de modo inconsistente en la bibliografía. El efecto persistente y la gravedad de los síntomas de TEP en un individuo se asocian con el combate. Yehuda et al. (1992) han encontrado que "atrocidad" y "exposición al combate" se relacionaron significativamente con los síntomas de reexperimentación (Buckley et al. 1998). Beckham et al. (1998) encontraron que la exposición al apoyo de atrocidades se relacionó con los síntomas de re-experimentación. El estudio de las respuestas antes y después de la muerte traumática de soldados que han participado en la "Operación Tormenta de Desierto" muestra que en la exposición posterior se incrementan significativamente los síntomas de intrusión para todos los grupos expuestos a la muerte. Se observó un aumento de los síntomas de evitación post-exposición en los dos grupos con mayor exposición a restos humanos. No hubo aumentos significativos en intrusión o evitación en el grupo de los no expuestos (McCarol et al. 2001). Los efectos del combate, de ser heridos y la disociación peritraumática han sido evaluados juntos en cada uno de los clusters de síntomas de TEP en un estudio de cohortes de una muestra al azar de veteranos australianos de Vietnam. Cada uno se relacionó de manera diferente con la re-experimentación, evitación, hiperactivación y diagnóstico de TEP (O'Toole et al. 1999). El número de experiencias de guerra es un predictor significativo en todos los clusters de TEP (Michultka et al. 1998). Las relaciones dosis-efecto entre las experiencias acumulativas y traumáticas y los síntomas psiquiátricos son positivas para la subcategoría del TEP de "incremento de la activación", que constituye la asociación más potente (Mollica et al. 1998).

Nuestros resultados demuestran que hay un alto efecto directo de la disociación peritraumática en los modelos causales de clusters de síntomas de activación postraumática, evitación e intrusividad. Actualmente está prestándose particular atención a los diferentes aspectos de la disociación en la formulación de modelos causales de TEP (Spiegel \& Cardena 1991, Brende 1987, Hyer et al. 1993, van der Kolk et al. 1996, Bremner \& Brett 1997, Marmar et al. 1994). Bremner y Brett (1997) documentaron que la incidencia de escenas retrospectivas ("flashbacks") se correlaciona con la disociación peritraumática. En una muestra de mujeres veteranas de Vietnam, la disociación peritraumática correlacionó con "intrusión” y " síntomas de evitación”, medidas mediante una encuesta, aunque estas relaciones no fueron confirmadas para un segundo índice de sintomatología de TEP. Se relacionaron los síntomas de evitación con un índice general de disociación (Tichenor et al. 1996). Tampke e Irwin (1999) mostraron que la ansiedad tenía un valor predictivo para los tres clusters de síntomas en los veteranos de guerra de Vietnam, pero los clusters se diferenciaban por el patrón de sus relaciones con las variables de disociación. Las variables de disociación de "ensimismamiento psicológico" y "disociación peritraumática" predecían los síntomas de activación (Tampke \& Irwin 1999). Los resultados de Feeny et al. (2000) son consistentes con la conceptualización de embotamiento como un rasgo nuclear del TEP crónico, pero estos resultados no son consistentes con los hallazgos de que las disociaciones peritraumáticas son predictivas de TEP. La falta de capacidad predictiva de la disociación para TEP tardío puede reflejar únicamente que esta relación es más compleja que 
aquélla existente entre otras variables (Feeny et al. 2000, Harvey \& Bryant 1998).

Nuestros resultados muestran que el recibimiento al regreso al hogar contribuye al desarrollo de clusters de síntomas postraumáticos con diferente influencia etiológica. Tiene el mayor efecto etiológico total entre las variables investigadas en el modelo para el cluster de síntomas "intrusividad" y tiene menor efecto total que las "experiencias en zona de guerra" y disociación en los otros dos modelos de clusters de evitación y de activación. La vuelta a casa y a la vida civil del ex-soldado es un acontecimiento crucial que puede determinar si la situación de tensión aguda permanecerá en intensidad subclínica o activará el desarrollo del TEP crónico (Fontana \& Rosenheck 1994, Vukšić-Mihaljević et al. 1998).

Según nuestro estudio, los factores de riesgo premilitares y las condiciones de entrada en el ejército juegan un papel causal secundario en los tres modelos, aunque en ocasiones es alto el efecto total de los factores de riesgo premilitares en el modelo causal del cluster de síntomas de evitación (los antecedentes de problemas psiquiátricos tienen el efecto total más alto) y lo mismo para las condiciones de entrada en el ejército en el modelo causal para el cluster de activación. Yehuda et al. (1998) han encontrado que había relaciones significativas entre los padres y sus descendientes adultos, atendiendo al efecto de un trauma en la propia vida y el nivel de intrusividad, pero no entre los síntomas de evitación como respuesta al recuerdo del holocausto. En un reciente estudio de sintomatología del TEP después de un terremoto, McMilen et al. (2000) han relacionado los antecedentes psiquiátricos personales con los síntomas de evitación e insensibilización.
Los resultados pueden apoyar la hipótesis de estudio de que todos los síntomas de TEP no tienen la misma etiología, y esa jerarquización de influencia etiológica diferente se da entre las variables estudiadas en los tres modelos desarrollados de clusters de síntomas de TEP. El resultado del porcentaje relativamente pequeño de clusters de síntomas de TEP descritos en los modelos construídos aviva el problema de la selección de variables para que sean integrada en un modelo y está estrechamente unido con el número limitado de variables que pueden evaluarse en los modelos.

\section{Bibliografía}

BECKHAM, J.C., FELDMAN, M.E., KIRBY, A.C. Atrocities exposure in Vietnam combat veterans with chronic postraumatic stress disorder: relationship to combat exposure, symptom severity, guilt, and interpersonal violence. Journal of Traumatic Stress 11(4), 777-85, 1998.

BLANCHARD, E.B., JONES-ALEXANDER, BUCKLEY T.C., FORNERIS, C.A. Psychometric properties of the PTSD Checklist (PCL). Behaviour Research and Therapy $34,669-673,1996$.

BOLLEN, K.A. Structural Equations With Latent Variables. New York: Wiley, 1989.

BREMNER, J.D., BRETT, E. Trauma-related dissociative states and long-term psychopathology in posttraumatic stress disorder. Journal of Traumatic Stress 10, 37-49, 1997.

BRENDE, J.O. Dissociative disorders in Vietnam combat veterans. Journal of Contemporary Psychotherapy 17, 77-86, 1987.

BRYANT, R.A., HARVEY, A.G. Initial posttraumatic stress responses following motor vehicle accidents. Journal of Traumatic Stress 9, 223-234, 1996.

BUCKLEY, T.C., BLANCHARD, E.B., HICKLING, E.J. A confirmatory factor analysis of posttraumatic stress symptoms. Behaviour Research and Therapy 36(11), 1091-9, 1998.

FEENY, N,C., ZOELLNER, L.A., FITZGIBBONS, L.A., FOA, E.B. Exploring the roles of emotional num- 
bing, depression, and dissociation in PTSD. Journal of Traumatic Stress 13(3), 489-498, 2000.

FIRST, M.B., SPITZER, R.L, GIBBON, M., WILLIAMS, J.B.W. Structured Clinical Interview for DSMIV-TR Axis I Disorders, Research Version, Non-patient Edition (SCID-I/NP). New York: Biometrics Research, New York State Psychiatric Institute, 2002.

FONTANA, A., ROSENHECK, R. A causal model of the etiology of war-related PTSD. Journal of Traumatic Stress 6, 475-500, 1993.

FONTANA, A., ROSENHECK, R. Posttraumatic stress disorder among Vietnam theater veterans: A causal model of etiology in a community sample. Journal of Nervous and Mental Disease 182, 677-684, 1994.

FONTANA, A., ROSENHECK, R. Attempted suicide among Vietnam veterans: A model of etiology in a community sample. American Journal of Psychiatry 152, 102$109,1995$.

HARVEY, A.G., BRYANT, R.A. The relationship between acute stress disorder and posttraumatic stress disorder: A prospective evaluation of motor accident survivors. Journal of Consulting and Clinical Psychology 66, 507$512,1998$.

HELZER, J.E., ROBINS, L.N., MCEVOY, L. Posttraumatic stress disorder in general population. New England Journal of Medicine 317, 1630-1634, 1987.

HELZER, J.E. Methodological issues in the interpretations of the consequences of extreme situations. In: Dohrenwend BS, Dohrenwend BP, ed. Stressful Life Events and Their Contexts. New York: Prodist, 1981.

HYER, L.A., ALBRECHT, J.W., BOUDEWYNS, P.A., WOODS, M.G., BRANDSMA, J. Dissociative experiences of Vietnam veterans with chronic posttraumatic stress disorder. Psychological Reports 73, 519-530, 1993.

KADUSHIN, C., BOULANGER, G., MARTIN, J. Legacies of Vietnam. Vol. IV. Long term stress reactions: Some causes, consequences, and naturally occuring support systems. Washington, DC: House Committee Print No. 14, U.S. Government Printing Office, 1981.

KING, D.W., KING, L.A. A brief introduction to structural equation modeling. PTSD Research Quaterly 8(4), 16, 1997.

KULKA, R.A., SCHLENGER, W.E,, FAIRBANK. J.A., HOUGH, R.L., JORDAN, B.K., MARMAR, C.R., WEISS, D.S. Trauma and the Vietnam war generation: Report of findings from the national Vietnam veterans readjustment study. New York: Brunner/Mazel, 1990.

LAUFER, R.S., BRETT, E., GALLOPS, M.S. Dimensions of posttraumatic stress disorder among Vietnam vete- rans. Journal of Nervous and Mental Disease 173, 538545,1985 .

LEE, K.A., VAILLANT, G.E., TORREY, W.C., ELDER, G.H. A 50-year prospective study of the psychological sequelae of World War II combat. American Journal of Psychiatry 152, 516-522, 1995.

MARMAR, C. Preliminary data. Personal communication. December 1989.

MARMAR, C.R., WEISS, D.S., SCHLENGER, W.E., FAIRBANK, J.A., JORDAN, B.K., KULKA, R.A., HOUGH, R.L. Peritraumatic dissociation and posttraumatic stress in male Vietnam theater veterans. American Journal of Psychiatry 151, 902-907, 1994.

McCAROLL, J.E., URSANO, R.J., FULLERTON, C.S., LIU, X., LUNDY, A. Effects of exposure to death in a war mortuary on posttraumatic stress disorder symptoms of intrusion and avoidance. Journal of Nervous and Mental Disease 189(1), 44-8, 2001.

McMILLEN, J.C., NORTH, C.S., SMITH, E.M. What parts of PTSD are normal: intrusion, avoidance, or arousal? Data from the Northlidge, California, earthquake. Journal of Traumatic Stress 13(1), 57-75, 2000.

MICHULTKA, D., BLANCHARD, E.B., KALOUS, T. Responses to civilian war experiences: predictors of psychological functioning and coping. Journal of Traumatic Stress 11(3), 571-577, 1998.

MOLLICA, R.F., McINNES, K., PHAM, T., SMITH , FAWZI, M.C., MURPHY, E., LIN, L. The dose-effect relationship between torture and psychiatric symptoms in Vietnamese ex-political detainees and a comparasion group. Journal of Nervous and Mental Disease 186(9), 543-553, 1998.

O'TOOLE, B.I., MARSHALL, R.P., SCHURECK, R.J., DOBSON, M. Combat, dissociation, and posttraumatic stress disorder in Australian Vietnam veterans. Journal of Traumatic Stress 12(4), 625-640, 1999.

RAUSCH, L., KNUTSON, J.F. The self-report of personal punitive childhood experiences and those of siblings. Child Abuse \& Neglect 15, 29-36, 1991.

SAS Institute Inc. SAS Technical Report P - 200, SAS / STAT Software: CALIS and LOGISTIC Procedures, Release 6.04. Cary. North Carolina: SAS Institute, 1990.

SPIEGEL, D., CARDENA, E. Disintegrated experience: the dissociative disorders revisited. Journal of Abnormal Psychology 100, 366-378, 1991.

TAMPKE, A.K., IRWIN, H.J. Dissociative processes and symptoms of posttraumatic stress in Vietnam Veterans. Journal of Traumatic Stress 12(4), 725-738, 1999. 
TICHENOR, V., MARMAR, C.R., WEISS, D.S., METZLER, T.J., RONFELDT, H.M. The relationship of peritraumatic dissociation and posttraumatic stress: Findings in female Vietnam theater veterans. Journal of Consulting and Clinical Psychology 64, 1054-1059, 1996.

VAN DER KOLK, B.A., VAN DER HART, O., MARMAR, C.R. Dissociation and information processing in posttraumatic stress disorder. In: van der Kolk BA, McFarlane AC, Weisaeth L., ed. Traumatic Stress: The effects of overwhelming experience on mind, body, and society. New York: Guillford Press, 1996.

VUKŠIĆ-MIHALJEVIĆ, Ž., MANDIĆ, N, BENŠIĆ,M., MIHALJEVIĆ, S. Posttraumatic stress disorder among Croatian veterans: a causal model. Psychology and Clinical Neuroscience 54(6), 625-636, 2000.

VUKŠIĆ-MIHALJEVIĆ, Ž., MANDIĆ, N., LAUFER, D., BARKIĆ, J., FILAKOVIĆ, P. Combat-related posttraumatic stress disorder and social functioning. The European Journal of Psychiatry 12, 225-231, 1998.

VUKŠIĆ-MIHALJEVIĆ, Ž., MANDIĆ, N., MIHALJEVIĆ, S., IVANDIĆ, A. Symptom structure and psychiatric comorbidity of combat-related post-traumatic stress disorder. Psychology and Clinical Neuroscience 53, 343-349, 1999.
WEATHERS, F.W., LITZ, B,T., HERMAN, D.S., HUSKA, J.A., KEANE, T.M. The PTSD checklist: reliability, validity and diagnostic utility. Paper presented at the Annual Meeting of the International Society for Traumatic Stress Studies. San Antonio, TX, October, 1993.

YEHUDA, R., SCHMEIDLER, J., GILLER EL, J.R., SIEVER, L.J., BINDER-BRYNES, K. Relationship between posttraumatic stress disorder characteristics of Holocaust survivors and their adult offspring. American Journal of Psychiatry 155(6), 841-843, 1998.

YEHUDA, R., SOUTHWICK, S.M., GILLER, E.L., J.R. Exposure to artrocities and severity of chronic posttraumatic stress disorder in Vietnam combat veterans. American Journal of Psychiatry 149(3), 333-336, 1992.

\footnotetext{
Dirección para correspondencia: Željka Vukšić-Mihaljević PhD Department of Psychiatry University Hospital Osijek University School of Medicine 4 Huttler Street 31000 Osijek e-mail mihaljevic.zeljka@kbo.hr CROATIA
} 